\title{
Unlocking Metamaterial Properties through Multiscale Design
}

\author{
Scott Townsend ${ }^{1, *}$, Shiwei Zhou ${ }^{2}$ and Qing $\operatorname{Li}^{1}$ \\ ${ }^{1}$ School of Aerospace, Mechanical and Mechatronic Engineering, The University of Sydney, Sydney, NSW 2006, Australia \\ ${ }^{2}$ School of Civil, Environmental and Chemical Engineering, RMIT University, GPO Box 2476, Melbourne 3001, Australia
}

\begin{abstract}
Extraordinary electromagnetic behaviour, such as negative refractive index, can be produced in metamaterial composites. A number of impressive specimens have been produced to date, though they typically rely on conductor inclusions with intricate topologies, or dielectric inclusions with very high permittivity. We show that by designing the composite on two different length scales, we are able to bring about metamaterial behaviour using quite simple constituent materials and topologies. One scale is analyzed in the long wavelength limit, where metallic (or otherwise conducting) nanoparticles are mixed into a common dielectric phase, producing material with a high, and tunable, permittivity. This material is then used as the inclusion in the larger scale, where the high permittivity produces electric and magnetic Mie resonances, leading to a negative refractive index of the composite as a whole.
\end{abstract}

Keywords: Multiscale Materials, Multiscale Modelling, Metamaterials

\section{Introduction to Negative Index Metamaterials}

Metamaterials are those composites which possess supernatural material properties. To date, metamaterials have been produced with such remarkable properties as negative Poisson's ratio [1], negative thermal expansion coefficient [2] and negative refractive index [3].

Negative refractive index (NRI) material has become a particularly salient research topic, as it can lead to the realization of a number of inspiring devices such as the invisibility cloak [4] (Fig. 2), light trap [5] and superlens [6]. The refractive index can become negative when at either of the material parameters $\epsilon$ (electric permittivity) or $\mu$

*Corresponding author: scott.townsend@sydney.edu.au
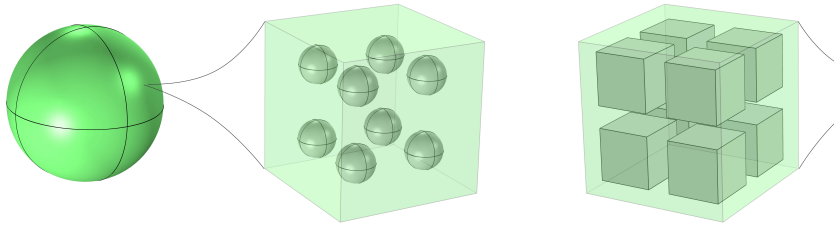

(a)

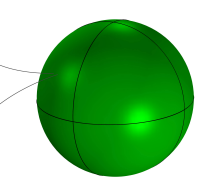

(b)

Figure 1: (a) Two sets of high-permittivity spheres are produced from a dielectric-conducting nanoparticle mixture; the permittivity value can be tuned by varying the volume fraction (and topology) of the nanoparticles (b) The spheres are dispersed in a host medium to create a slab of metamaterial which can possess a double-negative refractive index. 
(magnetic permeability) is less than zero. In fact, double-negative refractive index (DNRI) is preferred (achieved when both $\epsilon$ and $\mu$ are $<0$ ), since it allows for the design of matched and/or low-loss media [7].

A number of methods aimed at achieving DNRI behaviour have been proposed to date. All methods rely on creating a composite by dispersing inclusions into a host media; electric and magnetic resonances are simultaneously induced in the composite via judicious choice of the inclusion structure.

A lot of early research effort was directed towards split ring resonator-type metamaterials, in which metallic patterns are printed both sides of a dielectric substrate. Incident light induces current in the patterns, which can lead to DNRI behaviour [8]. So-called fishnet structures have also been proposed, which guide incident light through metallic apertures; careful selection of aperture shape can cause the composite to behave as DNRI material [9, 10].

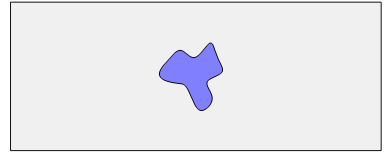

(a)

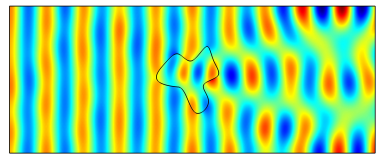

(b)

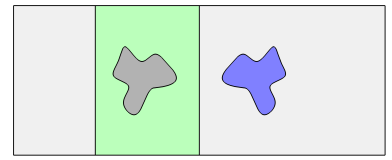

(c)

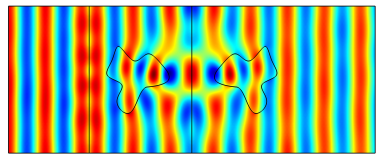

(d)

Figure 2: An invisibility cloak from double-negative index material: (a) An arbitrarily-shaped object is placed in a waveguide. (b) The object scatters an incident plane wave. (c) A slab with index $n=-1$ is placed in front of the object, along with the object's mirror-image. (d) The presence of the DNRI slab forces the incoming light waves to be transmitted and remain planar; the object is invisible.

Another route to DNRI behaviour is via the Mie resonance of small, traditionally spherical, particles. A particle with sufficiently high permittivity will undergo both electric and magnetic resonances, though for a homogeneous particle, the frequencies at which these resonances occur are separate [11]. In order to achieve simultaneous electric and magnetic resonance, it has been proposed to use sublattices of spheres of different size and/or material [12]; one lattice provides electric resonance, the other magnetic resonance.

It is this bi-lattice type of material which we will focus on, for a number of challenges remain before practical application can be found. Particularly, the requisite high-permittivity that the inclusions must possess greatly reduces the set of candidate materials that can be used. Materials such as BST [13] and $\mathrm{TiO}_{2}$ [14] have been proposed for frequencies up to the low THz; Tellurium [15] and Si [16] have been proposed for higher (optical) frequencies. The permittivity of the these materials cannot be easily tuned, which greatly lessens the designer's freedom when designing applications. In addition, losses are typically inherent in these materials (equating to absorption of incident light), which greatly detracts from the practicality.

Here we propose a multiscale design technique which may be capable of overcoming the above difficulties. We show that the requisite high-permittivity materials can be produced by dispersing metallic (or otherwise conducting) nanoparticles into a common dielectric phase. Moreover, the permittivity is highly tunable via the topology and volume fraction of the nanoparticles, which if sufficiently conductive, will also eliminate material losses. We propose to then use this high-permittivity material as spherical inclusions on a larger scale. Two sets of spherical inclusions will be designed - one targeting electric resonance, the other magnetic, such that a composite with double-negative refractive index can be realized.

\section{The Smaller Scale: Creating High-Permittivity Materials}

In this section we demonstrate a method of producing high-permittivity material from quite mundane constituents. The Clausius-Mosotti relation gives a concise description of the effective permittivity of a composite comprised of spherical inclusions with permittivity $\epsilon_{i}$ dispersed randomly in a host material with permittivity $\epsilon_{h}$ :

$$
\frac{\bar{\epsilon}-\epsilon_{h}}{\bar{\epsilon}+2 \epsilon_{h}}=\frac{f}{r^{3}} \alpha^{(\mathrm{E})}
$$

where $f, r$ and $\alpha^{(\mathrm{E})}$ are the volume fraction, radius and electric polarizability of the inclusions, respectively. Now provided $r$ is very small in comparison to the incident light wavelength, the electrostatic value of the polarizability can be used, and Eq. (1) reduces to the Maxwell-Garnett (M-G) formula:

$$
\bar{\epsilon}=\epsilon_{h}+3 f \epsilon_{h} \frac{\epsilon_{i}-\epsilon_{h}}{\epsilon_{i}+2 \epsilon_{h}-f\left(\epsilon_{i}-\epsilon_{h}\right)}
$$


The above has been shown to remain valid even up to very high values of $f$ [17], and although originally derived for spherical inclusions, we found via FEA simulation (using the theory of [18]) that Eq. (2) gives excellent predictions for all triple-symmetric topologies (cubes, etc.) as well. Now in the case of conducting inclusions, we have $\epsilon_{h} / \epsilon_{i} \rightarrow 0$, and so

$$
\bar{\epsilon} \underset{\epsilon_{h} / \epsilon_{i} \rightarrow 0}{=} \epsilon_{h} \frac{2 f+1}{1-f}
$$

For the M-G theory to remain valid for conducting inclusions, the dimension must also be smaller than the skin depth. In practice, then, the inclusions must be nanoparticles. As an aside, we note that it is quite easy to show [19] that Eq. (3) remains valid in the case that the inclusions are not solid conductors; instead they could be dielectric particles coated in a thin layer of conducting material, which may be a way to decrease the cost of these materials.

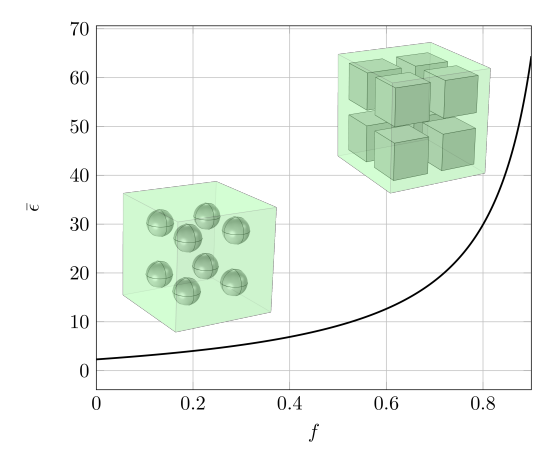

Figure 3: Effective permittivity of a dielectric-conductor nanoparticle composite.

Figure 3 shows the effective permittivity achieved for a host material $\epsilon_{h}=2.3$ (a typical dielectric) as the volume fraction of the conducting nanoparticles increases. As $f$ increases beyond 0.7, packing density dictates that it would no longer be possible to use nanospheres for the inclusion topology, and more complicated topology such as nanocubes, or even a number of different nanosphere sizes, would need to be utilised. We note that the effective permittivity is entirely real (there is no lossy component).

\section{The Larger Scale: Creating DNRI Material}

In this section, we show how the high-permittivity material from Sec. 2 can be used to induce DNRI on a larger scale. The technique is to manufacture two sets of high-permittivity spheres, and distribute them into a host material (as per Fig. 1). The Clausius-Mosotti formula from before can be re-written for the case of two sets of spherical inclusions [19]:

$$
\frac{\epsilon-\epsilon_{b}}{\epsilon+2 \epsilon_{b}}=\frac{v_{1}}{r_{1}^{3}} \alpha_{1}^{(\mathrm{E})}+\frac{v_{2}}{r_{2}^{3}} \alpha_{2}^{(\mathrm{E})}
$$

We used $\epsilon, \epsilon_{b}$ and $v$ in order to avoid confusion with the effective and host material permittivity, and volume fraction from Sec 2. Now in contrast to Sec. 2, we now assume that the inclusions are of sufficient size such that the electrostatic polarizability is no longer an adequate description. Doyle [20] suggested that the Mie theory could be utilised instead, such that

$$
\alpha^{(\mathrm{E})}=i \frac{3 r^{3}}{2 x^{3}} a_{1}
$$

where $x=\sqrt{\epsilon_{b}} k r$ is the size parameter of the spheres ( $k$ is the incident light wavenumber). The parameter $a_{1}$ is the first Mie coefficient, which in a scattering sense corresponds to the electric dipole mode. Now substituting Eq. (5) into Eq. (4) and rearranging, we get

$$
\epsilon=\epsilon^{\prime}+i \epsilon^{\prime \prime}=\epsilon_{b} \frac{1+3 i\left(\frac{v_{1} a_{1}^{(1)}}{x_{1}^{3}}+\frac{v_{2} a_{1}^{(2)}}{x_{2}^{3}}\right)}{1-\frac{3 i}{2}\left(\frac{v_{1} a_{1}^{(1)}}{x_{1}^{3}}+\frac{v_{2} a_{1}^{(2)}}{x_{2}^{3}}\right)} \quad \mu=\mu^{\prime}+i \mu^{\prime \prime}=\frac{1+3 i\left(\frac{v_{1} b_{1}^{(1)}}{x_{1}^{3}}+\frac{v_{2} b_{1}^{(2)}}{x_{2}^{3}}\right)}{1-\frac{3 i}{2}\left(\frac{v_{1} b_{1}^{(1)}}{x_{1}^{3}}+\frac{v_{2} b_{1}^{(2)}}{x_{2}^{3}}\right)}
$$


where the effective permeability $\mu$ was derived from a similar analysis, though this time for the magnetic polarization [21]; $b_{1}$ is the first Mie coefficient corresponding to the magnetic dipole mode. For the above to remain valid, the $x_{j}$ should remain low, as the theory assumes that the spheres act independently. The refractive index of the composite is retrieved as $n=n^{\prime}+i n^{\prime \prime}= \pm \sqrt{\epsilon \mu}$, with the requirement that for the passive materials considered, $n^{\prime \prime} \geq 0$.

As an illustrative example, consider two sets of spheres, each with $r=38 \mu \mathrm{m}$ and $v=0.25$, dispersed into a host medium with $\epsilon_{b}=1$. If the permittivities of the spheres are such that $\epsilon_{2}=15, \epsilon_{1}=29$, then it can be calculated that the first set will provide magnetic resonance at the target frequency of $1 \mathrm{THz}$; the second will provide electric resonance. Using Sec. 2, the required permittivities can be realized using the dielectric-conductor nanoparticle mixture proposed, with nanoparticle volume fractions of 0.65 (which can be realised with closely-packed nanospheres) and 0.79 (can be realised with, for example, nanocubes).

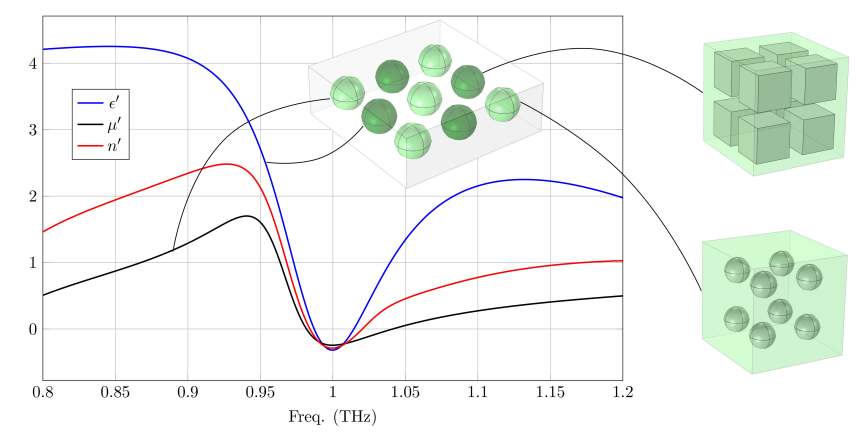

Figure 4: Frequency response of multiscale DNRI material. One set of spheres is designed for magnetic resonance at the target frequency; the other for electric resonance. The required sphere permittivity is realised by tuning the volume fraction in the dielectric-nanoparticle composites from Sec. 2

The simultaneous resonance afforded by the sphere lattice has produced a DNRI region at the desired frequency, demonstrating proof-of-concept for this design approach. Further research into the optimization and manufacturability of such composites will be conducted in the near future.

\section{References}

1. U.D. Larsen, O. Sigmund, and S. Bouwstra. In Micro Electro Mechanical Systems, 1996, MEMS'96, Proceedings. 'An Investigation of Micro Structures, Sensors, Actuators, Machines and Systems'. IEEE, The Ninth Annual International Workshop on, pages 365-371. IEEE, 1996.

2. O. Sigmund and S. Torquato. Journal of the Mechanics and Physics of Solids, 45(6):1037-1067, 1997.

3. David R Smith and Norman Kroll. Physical Review Letters, 85(14):2933, 2000.

4. Yun Lai, Huanyang Chen, Zhao-Qing Zhang, and CT Chan. Physical review letters, 102(9):093901, 2009.

5. Kosmas L Tsakmakidis, Allan D Boardman, and Ortwin Hess. Nature, 450(7168):397-401, 2007.

6. John Brian Pendry. Physical review letters, 85(18):3966, 2000.

7. Carlos García-Meca, Juan Hurtado, Javier Martí, Alejandro Martínez, Wayne Dickson, and Anatoly V Zayats. Physical review letters, 106(6):067402, 2011.

8. D.R. Smith, W.J. Padilla, DC Vier, S.C. Nemat-Nasser, and S. Schultz. Physical review letters, 84(18):4184-4187, 2000.

9. Gunnar Dolling, Martin Wegener, Costas M Soukoulis, and Stefan Linden. Optics Letters, 32(1):53-55, 2007.

10. Shiwei Zhou, Scott Townsend, Yi Min Xie, Xiaodong Huang, Jianhu Shen, and Qing Li. Optics Letters, 39(8):2415-2418, 2014.

11. Petr Chỳlek. JOSA A, 7(9):1609-1613, 1990.

12. Irina Vendik, Orest Vendik, and Mikhail Odit. Microwave and optical technology letters, 48(12):2553-2556, 2006.

13. Qian Zhao, Lei Kang, B Du, H Zhao, Q Xie, X Huang, B Li, J Zhou, and L Li. Physical review letters, 101(2):027402, 2008.

14. H Němec, C Kadlec, F Kadlec, P Kužel, Riad Yahiaoui, U-C Chung, Catherine Elissalde, Mario Maglione, and Patrick Mounaix. Applied Physics Letters, 100(6):061117, 2012.

15. James C Ginn, Igal Brener, David W Peters, Joel R Wendt, Jeffrey O Stevens, Paul F Hines, Lorena I Basilio, Larry K Warne, Jon F Ihlefeld, Paul G Clem, et al. Physical review letters, 108(9):097402, 2012.

16. DaJian Wu, ShuMin Jiang, Ying Cheng, and XiaoJun Liu. Optics express, 21(1):1076-1086, 2013.

17. Kimmo K Karkkainen, Ari Henrik Sihvola, and Keijo I Nikoskinen. Geoscience and Remote Sensing, IEEE Transactions on, 38(3):1303-1308, 2000.

18. Christian Brosseau and A Beroual. Progress in Materials Science, 48(5):373-456, 2003.

19. Ari H Sihvola. Electromagnetic mixing formulas and applications. Number 47. Iet, 1999.

20. William T Doyle. Physical review B, 39(14):9852, 1989.

21. Craig A Grimes and Dale M Grimes. Physical Review B, 43(13):10780, 1991. 